\title{
Position Statement
}

\section{USE OF AUTOMATED EXTERNAL DEFIBRILLATORS FOR CHILDREN ${ }^{1}$ ILCOR Advisory Statement, October 2002}

\section{BACKGROUND}

- Outside of a hospital setting, the use of an automated external defibrillator (AED) within the first 3 minutes of a witnessed adult ventricular fibrillation (VF) arrest results in survivor rates of greater than $50 \%$.

- For every one minute delay in defibrillation, the survival rate of a cardiac arrest victim decreases by 7 to $10 \%$.

- After more than 12 minutes of VF, the survival rate of adults is less than $5 \%$.

- In some settings (e.g. casinos, airport terminals) AED use has substantially improved the rate of survival from VF in adults.

- Trained responders have effectively used AEDs in many public settings, including casinos, airport terminals, and airplanes.

- VF is an uncommon cause of out-of-hospital pediatric cardiac arrest in infants (less than 1 year of age), but its occurrence increases with age.

- In-hospital studies of pediatric cardiopulmonary resuscitation (CPR) indicate that VF is not a rare rhythm among children in cardiac arrest.

- Previous recommendations limited AED use to children 8 years of age or older because of the concern that the minimum fixed energy level that AEDs deliver would be too high for smaller children and would damage the myocardium. A second concern was that the higher heart rate of children might be interpreted as VF by an AED and therefore shock inappropriately.

- Published reports and studies suggest that AEDs provide sensitive and specific rhythm analysis in infants and children.

- Because of increased thoracic impedance in young children, energy delivered to the heart may be lower than predicted.

- Limited data suggests that higher energy may be effective in children and does not cause myocardial damage.

- Pediatric cables which increase impedance and divert some energy away from the patient have been developed for the AED.

\section{RECOMMENDATIONS}

The Heart and Stroke Foundation of Canada recommends that:

1. Automated external defibrillators (AEDs) may be used for children 1 to 8 years of age with no signs of circulation. Ideally the device should deliver a pediatric-adjusted energy level but this is not a necessary requirement. The arrhythmia detection algorithm used in the device should demonstrate high specificity for pediatric shockable rhythms, i.e., it will not recommend delivery of a shock for nonshockable rhythms.

2. Every effort is made to confirm that the AED is safe when attached to and used on a child who does not have a shockable rhythm and who could be harmed by an inappropriate shock.

3. A lone rescuer responding to a child ( 1 to 8 years of age, less than $25 \mathrm{~kg}$ ) without signs of circulation activate the emergency medical services (EMS) system before any other action and provide 1 minute of CPR before attaching the AED.

4. Institutions that routinely care for children at risk for arrhythmias and cardiac arrest (e.g. in-hospital settings) continue to use defibrillators capable of energy adjustment for weight-based doses.

5. There is insufficient evidence for the Heart and Stroke Foundation of Canada to support a recommendation for or against the use of AEDs in children less than 1 year of age.

6. Hospitals and EMS systems that employ AEDs should develop specific guidelines for use of AEDs in children.

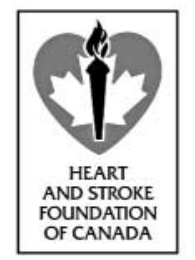

\section{REFERENCE}

1. Pediatric Advanced Life Support Task Force, International Liaison Committee on Resuscitation. Use of automated external defibrillators for children: an update. An advisory statement from the Pediatric Advanced Life Support Task Force, International Liaison Committee on Resuscitation. Circulation 2003:107;3250-5.

\section{The evidence contained in this Position Statement is current as of:}

